\title{
Observacion de deshidrataciones y contracciones de una arcilla por difracción de rayos x y microscopia electrónica a temperatura y humedad controladas
}

\author{
J. GARCIA-GUINEA(1), F. PITALUA (1), V. CORRECHER, (2), P. LÓPEZ-ARCE (3) \\ (1) Museo Nacional Ciencias Naturales, CSIC. Madrid, España. \\ (2) CIEMAT. 28500-Madrid. España. \\ (3) Centro Tecnológico de la Arcilla Cocida. AITEMIN. Toledo
}

\begin{abstract}
Se aplica una nueva puerta termo-refrigerada de construcción propia, con varios tipos de sensores acoplados (Análisis Térmico Diferencial, Humedad Relativa, Radioluminiscencia), a un difractómetro clásico para registrar perfiles de difracción secuenciales isotermales y noisotermales (en el rango de $17^{\circ} \mathrm{C}-200^{\circ} \mathrm{C}$ ). Con este dispositivo se estudiarán procesos de deshidratación y contracción de una arcilla histórica de Toledo. Además, se ha utilizado un microscopio electrónico ambiental (ESEM) con una cámara térmica interna $\left(\mathrm{de} 17^{\circ} \mathrm{C}\right.$ a $\left.1000^{\circ} \mathrm{C}\right)$ para secar la arcilla y analizarla con el EDS hasta $400^{\circ} \mathrm{C}$. En ambos equipos se controló la temperatura y la humedad relativa dentro de las cámaras de análisis. Los movimientos de contracción de secado han reorientado planos (001) de la illita, la muestra ha deshidratado hacia los $80^{\circ} \mathrm{C}$ y ha deshidroxilado hacia los $280^{\circ} \mathrm{C}$. A elevadas temperaturas in situ $\left(900^{\circ} \mathrm{C}\right)$, se tomaron fotografías de óptica electrónica del óxido de calcio procedente de la descomposición de la calcita.
\end{abstract}

Palabras clave: Illita, MEBA, DRX, deshidratación, deshidroxilación

Observation of dehydration and retrenchment effects in a claystone by $x$ ray diffraction and electron microscopy under control of temperature and relative humidity

A new designed stainless steel door, which holds cooling fluids, permits connect a set of sensors (DTA, RH, RL) to a traditional diffractometer and record sets of isothermal and non-isothermal diffraction profiles (in a range of $17^{\circ} \mathrm{C}-200^{\circ} \mathrm{C}$ ) allowing dehydration and retrenchment effects of an historical claystone from Toledo to be studied. In addition, a little oven into an environmental scanning electron microscope (ESEM) has been used to dry the claystone sample being analyzed with EDS up to $400^{\circ} \mathrm{C}$. In both equipments (XRD \& ESEM) chambers, temperature and relative humidity are controlled. The crystallographic (001) planes of illite are twisted during the water leaking. The dehydration (circa $80^{\circ} \mathrm{C}$ ) and dehydroxilation (circa $380^{\circ} \mathrm{C}$ ) processes of the illite are observed. At high temperature $\left(900^{\circ} \mathrm{C}\right.$ ) electron microscopy photographs of calcium oxide, stemming from calcite, were saved.

Keywords: Illite, ESEM, XRD, dehydration, dehydroxilation,

\section{INTRODUCCION}

Las contracciones experimentadas por la arcilla durante el secado y cocción de la cerámica roja dan origen a problemas de fisuras en productos acabados que conviene volver estudiar con nuevas técnicas $(1,2)$. Parte de estas roturas tienen su origen en indeseadas orientaciones de láminas de arcilla, durante el amasado y extrusión en fábrica, o durante el moldeo de piezas artesanales. Después de hacer varios estudios previos de correlación materia prima ladrillo, por métodos arqueológicos, mineralógicos, geoquímicos y experimentales, la arcilla propuesta para este estudio fue seleccionada por ser representativa de la materia prima original de los ladrillos históricos del casco medieval de Toledo. Dentro de la cámara de difracción de rayos $\mathrm{X}$, el control térmico a bajas temperaturas y el control de humedad ambiental, durante los análisis cinéticos secuenciales de secado permiten conocer movimientos de partículas. Por ejemplo, difractando monobloques de illita-pizarra a $80^{\circ} \mathrm{C}$, se registraron grandes deformaciones del pico $10.04 \AA \AA$ (001) de la illita atribuidos a procesos Ostwald-Ripening de disolución-precipitación de cationes potásicos movilizados por fluidos de deshidratación (3). El nuevo ESEM Phi- lips XL30/40 utiliza un detector de electrones secundarios gaseosos que emplea moléculas de agua dentro de la cámara para ampliar la señal, por lo que no es necesario metalizar la muestra para hacerla conductora a los electrones (4). El control de la temperatura dentro de la cámara del ESEM se realiza con horno eléctrico interno que alcanza $\operatorname{los} 1000^{\circ} \mathrm{C}$. En este trabajo se utilizan ambos equipos (DRX y ESEM) para observar las deshidrataciones y contracciones de una arcilla a temperatura y humedad controladas.

\section{PROCEDIMIENTO EXPERIMENTAL}

La muestra de arcilla.- Las muestras se tomaron en las proximidades del monasterio de San Bernardo para utilizar las mismas arcillas con las que se fabricaron los ladrillos históricos de Toledo. La información sobre las antiguas tejeras y ladrilleras se obtuvo de las Ordenanzas Municipales sobre el Ladrillo y Teja (títulos 87 y 86, desde el siglo XVII al XIX) en el Archivo Municipal de Toledo 
y en el Archivo Histórico Provincial y se comprobó por fotografía aérea (5). La determinación semicuantitativa de las fases mineralógicas de la muestra se realizó por difracción de rayos $\mathrm{X}$ con polvo desorientado (6), agregados orientados naturales, tratados con etilen-glicol y calentados a $550^{\circ} \mathrm{C}$ en un difractómetro Philips PW1710/00, (30Kv, 25mA), usando radiación $\mathrm{CuK}_{\alpha}(=1.54054 \AA)$. Los difractogramas fueron obtenidos desde $2^{\circ}$ hasta $60^{\circ} 2 \square$, en saltos de $0.05^{\circ}$ y con 2 segundos por escalón. Los análisis químicos puntuales se realizaron por energías dispersivas en el microscopio electrónico de barrido ambiental ESEM-Philips-XL30. El análisis térmico diferencial de la arcilla se realizó en un analizador térmico simultaneo ATD-TG-DSC (Setaram, Labsys CS 32-CS 332 Controller) a $10^{\circ} \mathrm{C} /$ minuto.

El montaje experimental en la cámara de DRX.- Mientras se realizan secuencias de perfiles de DRX, se controla la temperatura de la muestra (hasta $200^{\circ} \mathrm{C}$ ) y la humedad relativa dentro de la cámara, para ello, los autores han construido una nueva puerta original de acero en el Museo Nacional de Ciencias Naturales (7). La nueva puerta consta de los siguientes elementos: (a) Nuevo refrigerador de agua $\left(17^{\circ} \mathrm{C}\right)$, (b) Nueva puerta de acero hueca para alojar fluidos a diferentes temperaturas, (c) Calentadores de muestra dentro de la cámara (electrodo cerámico interno y lámpara halógena externa), (e) Alojamiento para la fibra óptica del espectrómetro CCD, (f) Nuevo circuito electrónico y nuevo software original para recoger análisis térmicos diferenciales entre muestra y patrón de referencia. (g) Humidificador que bombea vapor de agua dentro de la cámara, (h) Sensor de medición de humedad relativa dentro de la cámara de rayos X. (i) Sensor de medición de humedad relativa dentro de la cámara de rayos X. El controlador térmico (Eurotherm 2216) y la unidad de tiristores (Eurotherm TE10A) alimentan el electrodo cerámico o la lámpara halógena bajo control PID.

Accesorios de medición electrónica: Análisis térmico diferencial (ATD), radioluminiscencia (RL) y humedad relativa (HR).- Ambos termopares están conectados a un nuevo circuito de 22 bits (Analog Devices AD7710 ADC) conectado a un microcontrolador PIC16F84 de Microchip Technology Inc $(\mu \mathrm{C})$ que exporta los datos desde el ADC a un puerto serie. Un circuito auxiliar MAX232 modifica los niveles de salida del standard CMOS/RS232. El sistema permite medir las diferencias de temperaturas entre la muestra que se está difractando-calentado y la muestra patrón (meta-caolinita), el sistema electrónico mide 50 temperaturas por segundo con un programa original (Tambor) que es el mismo que se utiliza para registrar terremotos 24 horas al día. En la difracción se utiliza un tubo de foco ancho (Philips Cu PW2253/20) para conseguir una gran área de impacto $(12 \mathrm{~mm} \times 6 \mathrm{~mm}$ ) y una mayor emisión radioluminiscente (en el rango espectral de $190 \mathrm{~nm}$ a $850 \mathrm{~nm}$ ), recogida por fibra óptica y registra en un espectrómetro Ocean Optics S2000 CCD. En el lugar de la fibra óptica se puede instalar a traves de la puerta de acero un sensor de humedad (Digitron-2000 RH meter de Sifam Instruments). (Fig. 1a). El control térmico de la nueva puerta de acero se lleva a cabo con un programa Itools v.2.09 que también acciona una electro-válvula de cierre del líquido refrigerante.

El control de temperatura y humedad dentro del Scanning.- Se ha utilizado el nuevo microscopio electrónico de barrido ambiental ESEM (Philips XL 30) de la Universidad Juan Carlos I de Madrid, Este equipo permite trabajar a bajas presiones ( 2 torr en nuestro ensayo) y controlar las condiciones ambientales de temperatura (desde $17^{\circ} \mathrm{C}$ hasta $1000^{\circ} \mathrm{C}$ ) y humedad relativa, gracias a la utilización opcional de vapor de agua como atmósfera circulante. El sistema esta compuesto por un módulo de calentamiento (PW6752/31 Lab \& W y PW6752/32 FEG), un controlador de temperatura (PW67539) y una unidad de tiristores (PW6762/30) bajo control PID (8).
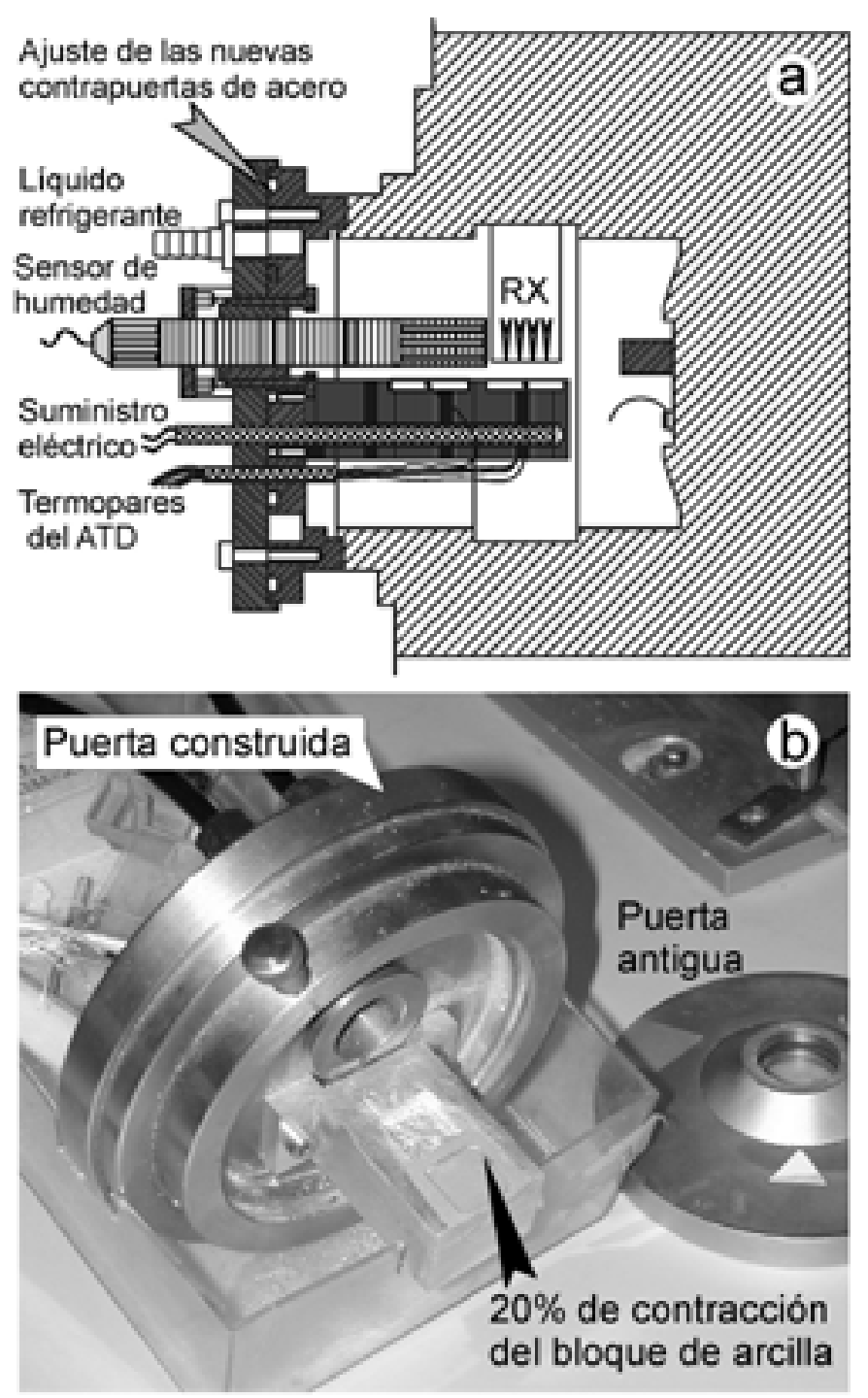

Figura 1.- Detalles del nuevo montaje experimental utilizado para el registro secuencias de difractogramas con control de temperatura y humedad relativa.

\section{RESULTADOS Y DISCUSIÓN}

La determinación semicuantitativa de los minerales de la muestra se realizó por difracción de rayos $\mathrm{X}$ (polvo desorientado), con el siguiente resultado: $35 \%$ filosilicatos, $42 \%$ cuarzo, $15 \%$ calcita, $3 \%$ dolomita, 5\% feldespatos (6). El agregado orientado de una fracción a $0.065 \mathrm{~m}$ potenció las reflexiones (001), pero después del tratamiento con etilen-glicol no mostró esmectitas, mientras que el calentamiento del agregado orientado a $550^{\circ} \mathrm{C}$ deshidroxiló la caolinita hacia metacaolinita por lo que la muestra aproximadamente tiene un $20 \%$ de moscovita, un 15\% de caolinita e indicios de clorita-clinocloro (Figura 2). Los análisis químicos puntuales por ESEM-EDS son congruentes con la composición mineralógica de las fases determinadas por difracción de rayos $\mathrm{X}$, por ejemplo: oxígeno $40.47 \%$, silicio $26.41 \%$, aluminio $12.67 \%$, calcio $8.24 \%$, hierro $6.29 \%$, potasio $3.57 \%$, magnesio $2.35 \%$. El análisis térmico diferencial de la arcilla mostró dos picos endotérmicos, uno correspondiente a la deshidratación a $80^{\circ} \mathrm{C}$, bien definido y otro mas plano correspondiente a una lenta deshidroxilación de los 


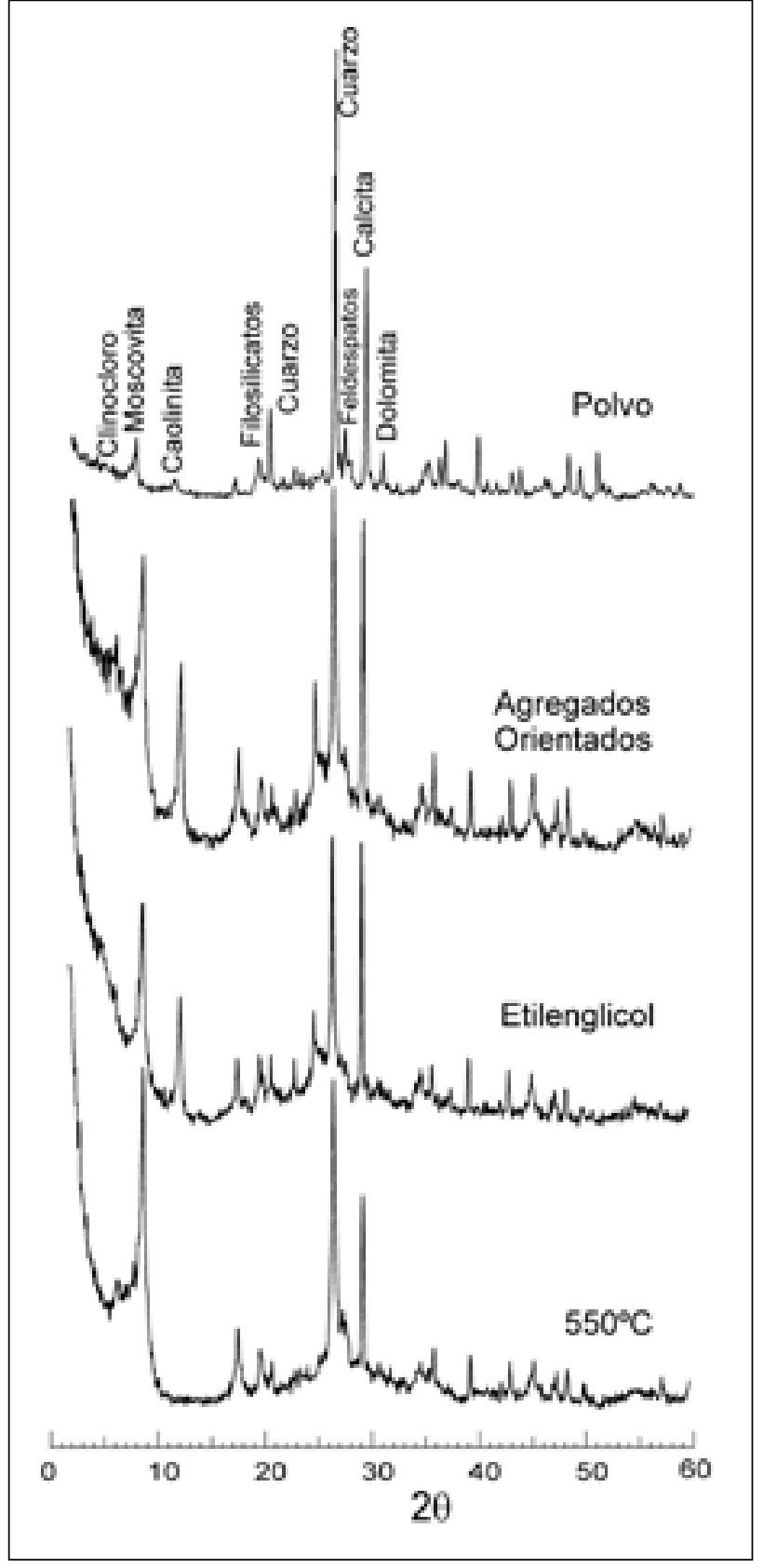

Figura 2.- Identificación de las fases mineralógicas de la muestra de arcilla por difracción de rayos a temperatura ambiente.

filosilicatos hacia los $280^{\circ} \mathrm{C}$. También se realizó un ATD in situ simultáneo para comprobar los procesos de deshidratación por debajo de los $100^{\circ} \mathrm{C}$, sin embargo, por la rotación del goniómetro, la curva de ATD resultante tiene un fondo de vibración bastante elevado y no se incluye en el trabajo.

La figura 3 muestra una secuencia no-isotermal de perfiles de tiempo-temperatura recogida por un programa original (SIRDAT) elaborado por el Dr. Martín-Ramos. Los perfiles fueron grabados secuencialmente en formato Rietweld y acumulados en diferentes tipos de matrices numéricas por otro programa original de Martín-Ramos (Pila.exe en Gwbasic). Finalmente, las matrices fueron procesadas con

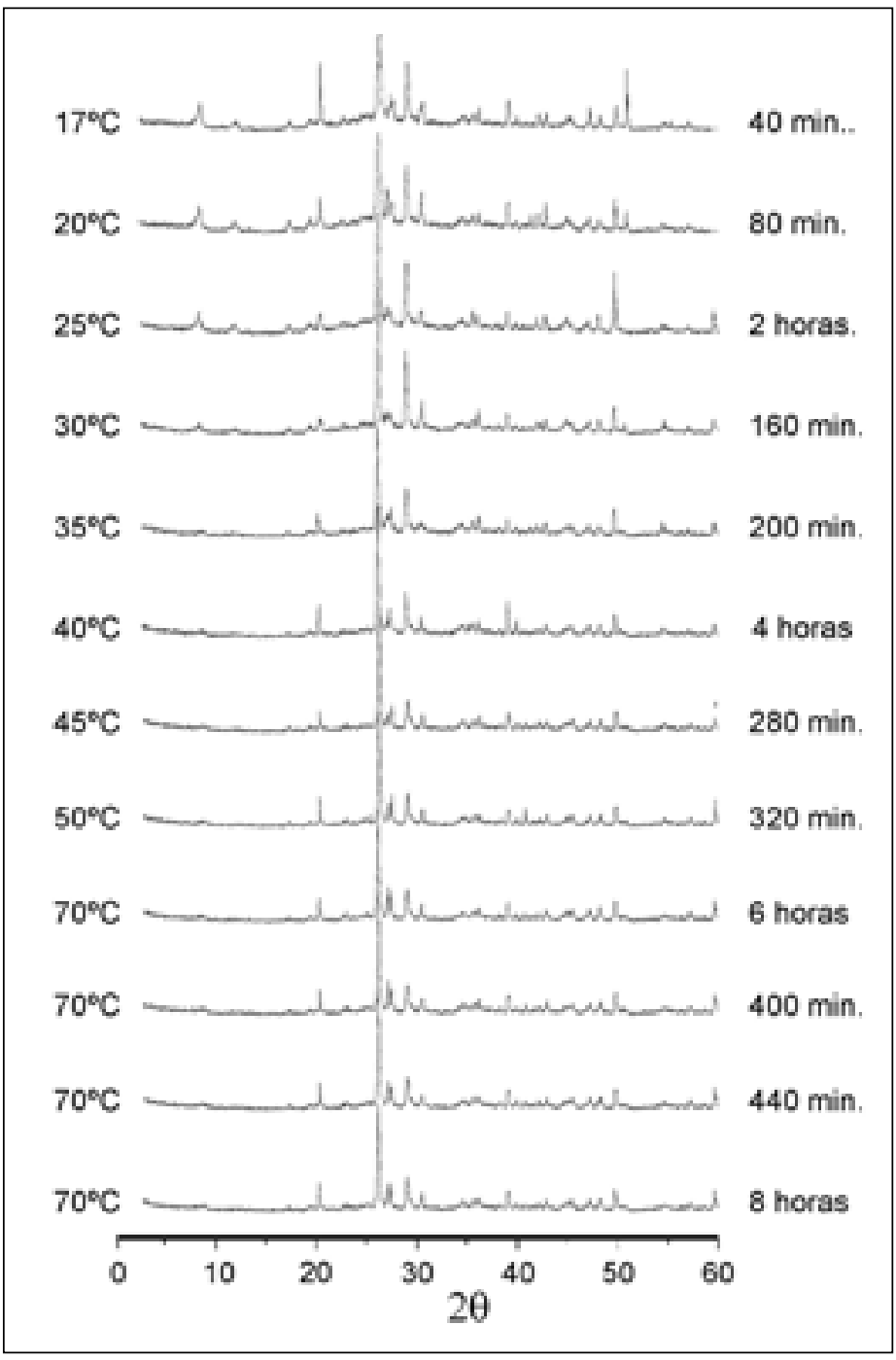

Figura 3.- Secuencia no isotermal de difractogramas de la arcilla imitando un proceso natural de secado de ladrillo de era bajo un día soleado.

un programa Origin. El ensayo recogió perfiles de 40 minutos a $17^{\circ} \mathrm{C}$, $20^{\circ} \mathrm{C}, 25^{\circ} \mathrm{C}, 30^{\circ} \mathrm{C}, 35^{\circ} \mathrm{C}, 40^{\circ} \mathrm{C}, 45^{\circ} \mathrm{C}, 50^{\circ} \mathrm{C}, 70^{\circ} \mathrm{C}$ para simular un proceso clásico de secado solar de bizcochos de ladrillo en una era. Se comprobó que trabajando en ambiente normal de aire seco, el sensor de humedad relativa deja de medir hacia $\operatorname{los} 65^{\circ} \mathrm{C}$ por desaparecer el vapor de agua del sistema. El resultado muestra una progresiva desaparición de las reflexiones basales de los filosilicatos (001) con el incremento de la temperatura-tiempo. Esta observación experimental se explica por varios fenómenos físicos simultáneos: (a) contracción de secado del bizcocho arcilloso, que reduce la superficie expuesta a los rayos X (Fig 1b) y (b) por desorientaciones espaciales de las láminas de arcilla por salida de agua a través de los microporos, esta última observación está avalada por la estabilidad térmica del pico $4.26 \AA ̊$ del cuarzo frente a la práctica desaparición del pico $10.04 \AA ̊$ de la illita.

El horno interno del ESEM permitió fotografiar partículas e interfases hasta $1000^{\circ} \mathrm{C}$ pero solo se pudo utilizar la sonda EDS hasta los $400^{\circ} \mathrm{C}$; temperatura a la que hubo que retirarlo. La contracción in situ produjo fuertes desplazamientos de la imagen hasta los $100^{\circ} \mathrm{C}$ (figu- 

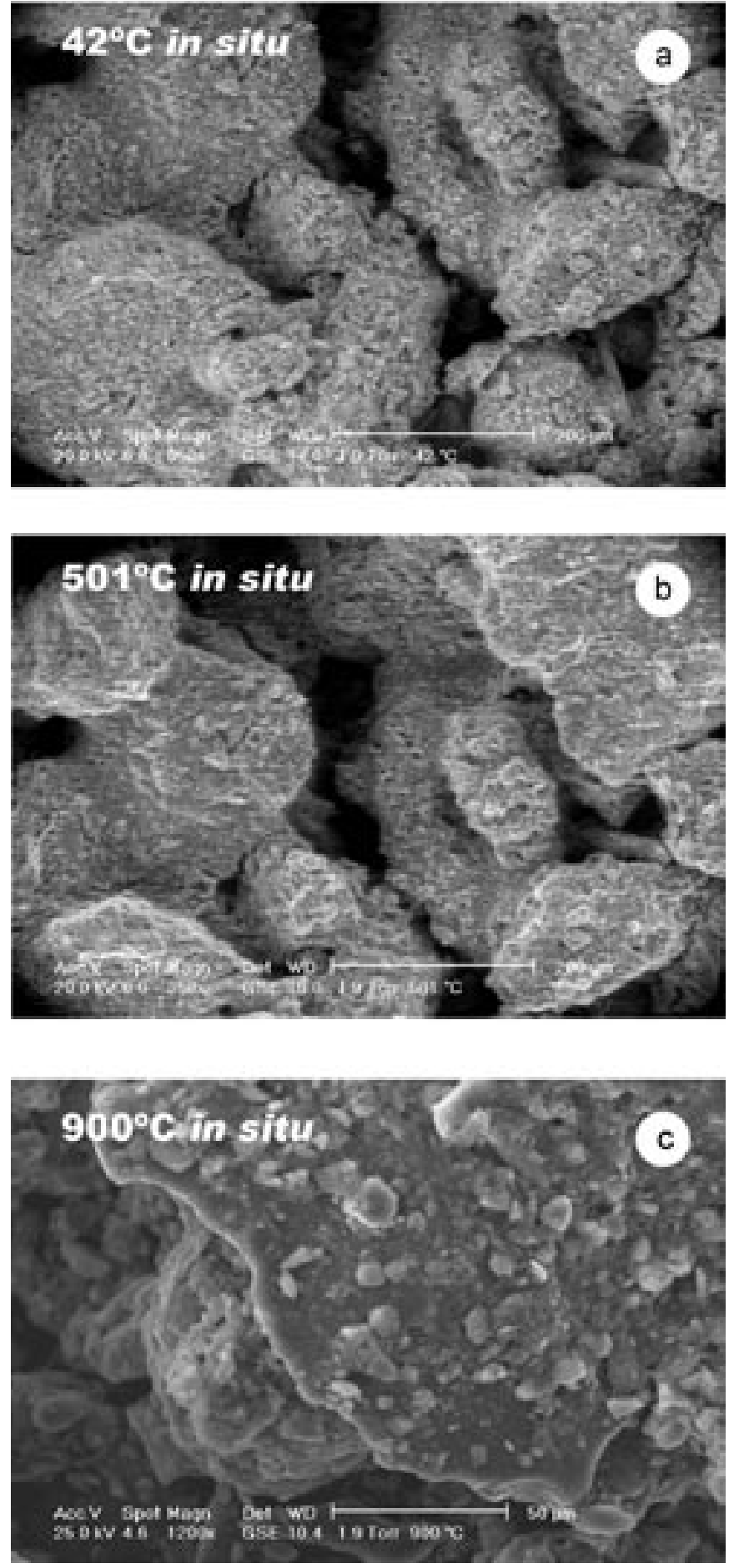

Figura 4.- Arcilla bajo el microscopio electrónico de barrido ambiental (con controles de humedad y temperatura). La calcita presente en la muestra calcinó por encima de $\operatorname{los} 750^{\circ} \mathrm{C}$. ra 4a). Hacia los $400^{\circ} \mathrm{C}-500^{\circ} \mathrm{C}$ (figura $4 \mathrm{~b}$ ) se volvieron a producir los mismos desplazamientos, atribuibles a la propia deshidroxilación de la illita. A altas temperaturas $\left(900^{\circ} \mathrm{C}\right)$ (figura $\left.4 \mathrm{c}\right)$ se observaron grietas en los gránulos de caliza debidos a la descomposición térmica de la calcita $\left(\mathrm{Ca} \mathrm{CO}_{3}+\mathrm{Q}->\mathrm{CO}_{2}+\mathrm{CaO}\right)$.

\section{AGRADECIMIENTOS}

Este trabajo ha sido financiado por el proyecto BFM2002-00048 de la CICYT.

\section{BIBLIOGRAFIA}

1. West-Richard. "Dilatometry of structural clay products". Canadian Ceramic Soc. Jour.. 34; Pages 29-33. (1965).

2. A.A. Diaz, R. Torrecillas. "Arcillas Cerámicas: Una revisión de sus distintos tipos, significados y aplicaciones". Bol. Soc. Esp. Ceram. V. 41 [5] 459-470 (2002).

3. J.Garcia-Guinea, V. Cardenes, V. Correcher, A. Delgado, M. Lombardero, J. C. Barros. "Deshidroxilaciones y efectos Ostwald ripening en pizarras de techar". Bol. Soc. Esp. Ceram. V. 39 [4], 589-594 (2002).

4. R. Combes, M. Robins, G. Blabier, M. Aidan, F. Degreve. “Visualization of imbibition in porous media by environmental scanning electron microscopy: application to reservoir rocks". Journal of Petroleum Science and Engineering 20 133-139 (1998).

5. Ministerio de Defensa. Fotografías aéreas. Vuelo General de España E. 1: 18.000. Pasada F, Hoja 629. N 6-7 y 11-12 (1985).

6. L. G. Schultz. "Quantitative interpretation of mineralogical composition from X-ray and chemical data for the Pierre Shale". U. S: Geol. Survay Prof. (1964). Paper, 391 C.

7. J. Garcia-Guinea, R. Ortiz, V. Correcher, A. La Iglesia, J. D. Martín-Ramos. "Improvements to X-ray diffractometers: Heaters, hygrometry and thermodiferential and spectra analyses". Review of Scientific Instruments 72, no. 10, (2001) 4005-4007.

8. P. Cova, F. Fantini. "On the effect of power cycling stress on IGBT modules". Microelectronic and Reliability, (1998), v. 38, 1347-1352 (1998).

Recibido: 01.02 .03

Aceptado: 30.11 .03 